CASE REPORT

Volume 16 Issue 12021

DOI: $10.21315 /$ aos2021.16.1.9

ARTICLE INFO

Submitted: $27 / 8 / 2020$

Accepted: 26/1/2021

Online: 25/6/2021

\section{Role of Speech Aid Prosthesis as Diagnostic and Therapeutic Aid for Velopharyngeal Insufficiency Defect: A Case Report}

\author{
Asikul Wadud ${ }^{\mathrm{a}}$, Waqas Tanveer ${ }^{\mathrm{b} *}$, Natdhanai Chotprasert ${ }^{\mathrm{c}}$ \\ Theerathavaj Srithavajc
}

${ }^{a}$ Department of Prosthodontics, Update Dental College E Hospital, Dhaka University, Dhaka-1711, Bangladesh

${ }^{b}$ Department of Oral and Maxillofacial Surgery, VU University Medical Center, 1007 MB Amsterdam, The Netherlands

${ }^{c}$ Maxillofacial Prosthetics Unit, Department of Prosthodontics, Faculty of Dentistry, Mahidol University, Bangkok 10400, Thailand

*Corresponding author: waqas_tanveer30@hotmail.com

How to cite this article: Wadud A, Tanveer W, Chotprasert N, Srithavaj T (2021). Role of speech aid prosthesis as diagnostic and therapeutic aid for velopharyngeal insufficiency defect: A case report. Arch Orofac Sci, 16(1): 87-94. https://doi.org/10.21315/aos2021.16.1.9

To link to this article: https://doi.org/10.21315/aos2021.16.1.9

\begin{abstract}
Soft palate tumors pose the challenge during reconstructive and rehabilitating procedures. Surgical resection of these tumors leads to velopharyngeal insufficiency (VPI). The primary effects of VPI are hypernasality and air-flow escape, while the secondary effects are abnormalities in speech articulation. Surgical revision along with speech therapy is a common approach to the treatment of VPI. Prosthetic management by means of speech aid prosthesis helps to reduce resonance, nasal emission and consonants errors. This clinical report describes the different stages of rehabilitation of velopharyngeal insufficiency defect following resection of malignant melanoma of left posterior alveolar ridge and soft palate. The speech aid prosthesis helped to rehabilitate the velopharyngeal insufficiency defect and aided in the diagnosis of extent of speech function improvement by perceptual and objective methods.
\end{abstract}

Keywords: Hypernasality; nasalance score; nasometer; speech aid prosthesis; velopharyngeal insufficiency

\section{INTRODUCTION}

The soft palate along with posterior and lateral pharyngeal walls establishes velopharyngeal (VP) closure. However, resection of soft palate due to tumors of tonsils, soft palate or posterior pharyngeal wall adversely affects this VP closure mechanism due to velopharyngeal insufficiency (VPI) (Curtis \& Beumer, 1996). The diagnosis of VP insufficiency can be made with perceptual speech evaluation, multi-view video fluoroscopy (MVF), nasopharyngoscopy (Seagle et al., 2002; Pegoraro-Krook et al., 2008; Sie et al., 2008) and magnetic resonance imaging (MRI) (Atik et al., 2008; Kao et al., 2008). Surgical correction and prosthetic rehabilitation are the mainstay procedures for treatment of VPI. There are various surgical options available for management of VPI including pharyngeal flaps and pharyngoplasty surgeries (Meek et al., 2003; Sipp et al., 2008; Van Lierde et al., 2008; Mink van der Molen et al., 2009). However, if surgical procedures are deemed unsuitable then 
prosthetic rehabilitation can be an alternative step, as speech aid prosthesis can alleviate functional deficiency up to nearly normal level. Main objective of speech aid prosthesis is to obturate the soft palate defect while speaking and swallowing to prevent nasal emission of air and direct the food to the esophagus (Tuna et al., 2010).

Resonance is the feature of voice that is distinguished by the balance of sound vibration in the nasal, oral and pharyngeal cavities during speech. Abnormal resonance can develop if there is blockage or obstruction in one of the following cavities, promoting hyponasality or cul-desac resonance, or if there is velopharyngeal dysfunction (VPD), can lead to hypernasality and/or nasal emission.

Nasal emission of air causes hypernasality and hinders speech articulation. Therefore functional molding is necessary to allow the lateral and posterior pharyngeal muscles to constrict and contact the bulb portion of the speech aid prosthesis during function. Following prosthetic rehabilitation, the role of speech therapist is important in resolving speech articulation errors and assessment of speech by various subjective tools. This case report describes the role of speech aid prosthesis as a therapeutic and diagnostic tool during rehabilitation of velopharyngeal insufficiency defects.

\section{CASE REPORT}

A Thai female patient, aged 38 years, had a history of malignant Melanoma on the left posterior alveolar ridge and soft palate. After surgical resection, the skin graft was placed on the upper left lateral wall of defect (Fig. 1 and Fig. 2). Prosthetic management was carried out in three stages; immediate prosthesis after surgical resection of tumor, interim prosthesis, after two months and definitive prosthesis after six months of surgical resection.

An alginate impression was made prior to the planned surgery to fabricate immediate surgical obturator with heat cure acrylic. This immediate surgical obturator was inserted immediately following tumor resection after some adjustments and tissue conditioner (GC America Inc, USA) relining to cope up with progressive healing process. Immediate
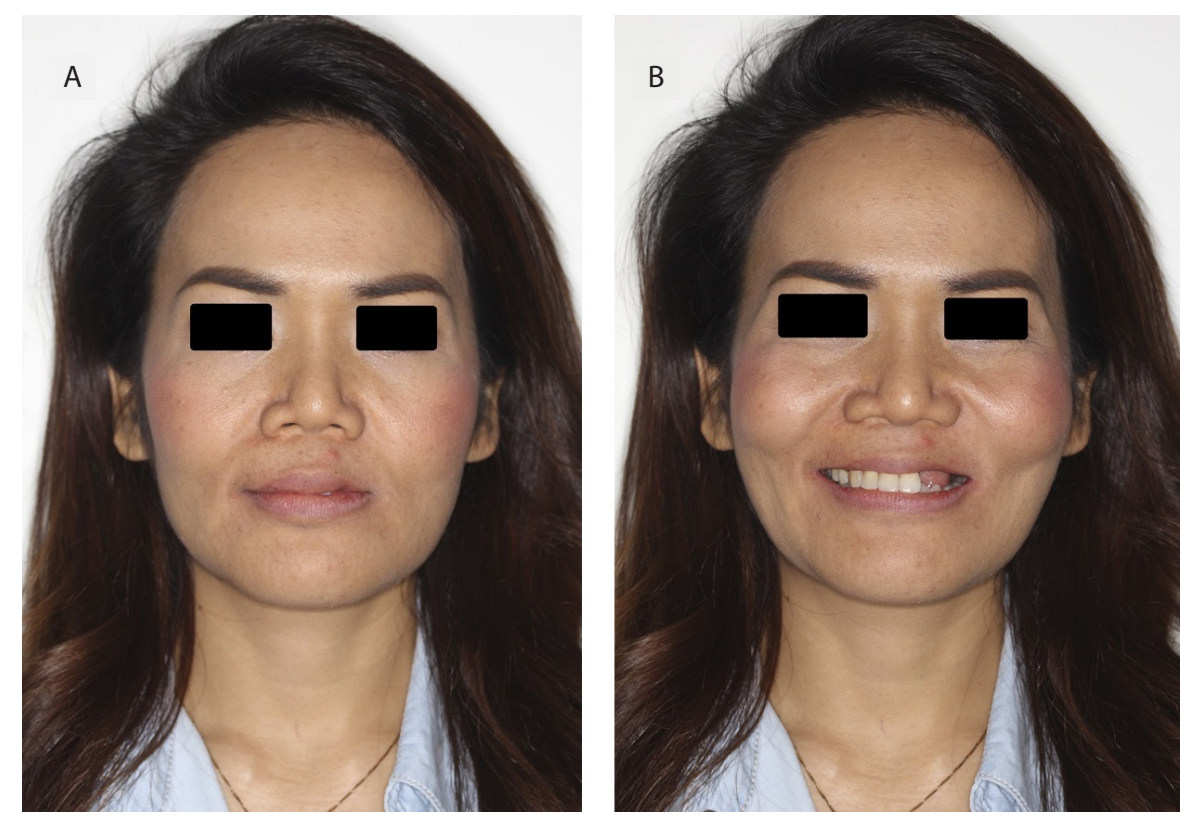

Fig. 1 Extraoral presentation without prosthesis. (A) The left side of the lip was depressing inward.

(B) Missing left maxillary teeth and inadequate lip support. 
surgical obturator was replaced with a delayed surgical obturator after two weeks and relined with tissue conditioner in the speech bulb area by functional moulding.

After two months of surgery, interim speech aid prosthesis was fabricated with heat cure acrylic. Missing teeth \# 22-25 were added in interim speech aid prosthesis and the speech bulb was extended to improve speech and deglutition. Functional moulding was done by using a tissue conditioner. Perceptual assessment was done by the speech therapist and the obturator bulb area was adjusted accordingly (Fig. 3). After a satisfactory perceptual assessment report, the objective method of assessment was adopted by analysing speech components using a nasometer. The patient was instructed to read oral and nasal Thai passages while the nasometer recorded the quality of speech. The speech was assessed according to various parameters including speech intelligibility, resonance, nasal airflow, consonant errors, grimace, voice, language and nasalance scores (Table 1). The reports had shown improved quality of speech when rehabilitated with interim prosthesis as compared to without prosthesis. The nasalance score had reduced from $74 \%$ to $57 \%$ after interim prosthesis (Table 1 ).

The interim speech aid prosthesis was followed by fabrication of definitive speech aid prosthesis after six months (Fig. 4). The definitive speech aid prosthesis was fabricated by following removable partial denture (RPD) principles. The bulb area on the master cast was blocked out with plaster and pumice mixture to guide the metal extension at the palatal plane. Interim speech aid prosthesis bulb portion was copied and pattern was transferred to definitive metal framework, which was further modified by
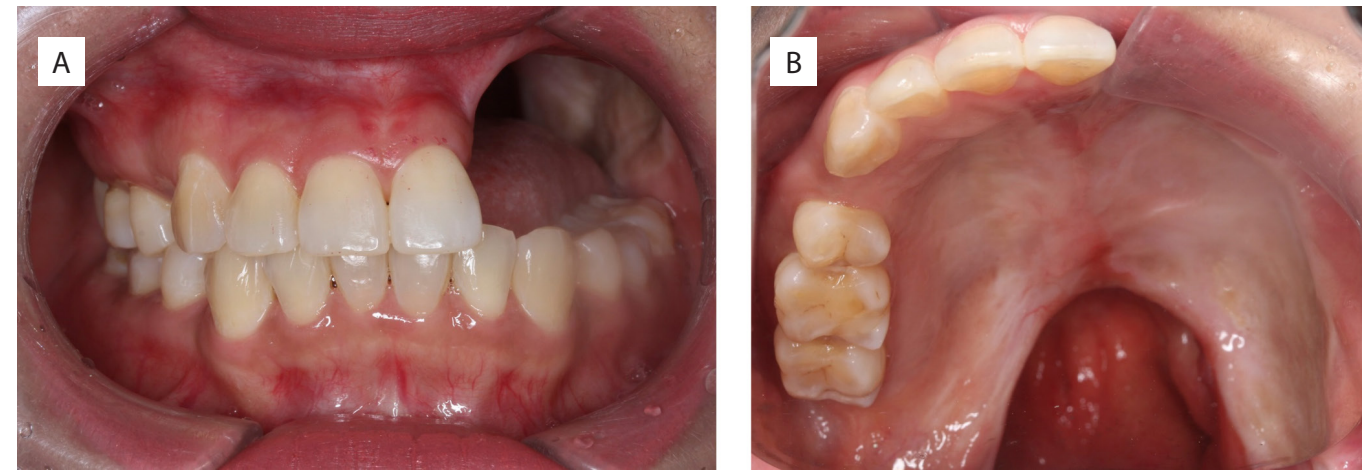

Fig. 2 Intraoral presentation without prosthesis. (A) Absence of left maxillary teeth and associated hard and soft tissue. (B) Extent of velopharyngeal insufficiency defect.

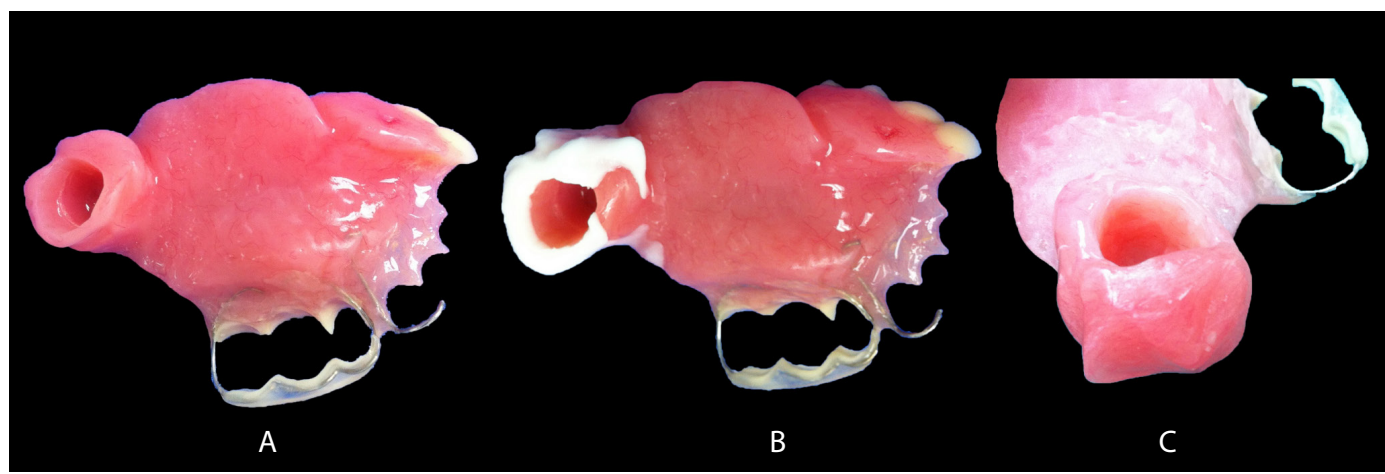

Fig. 3 Interim speech aid prosthesis. (A) Interim obturator. (B) Bulb portion relined with tissue conditioner. (C) Bulb portion relined with heat cure acrylic. 
tissue conditioner lining to extend $3 \mathrm{~mm}$ horizontally and vertically by functional moulding. Functional molding was done through functional movements by moving the head vertically; upwards and downwards, horizontally; right side to left side and mixture of movements by moving the head downwards to touch the chin with chest and move right side to the left side (Fig. 5). The bulb portion of speech aid prosthesis was relined until a satisfactory outcome was attained on perceptual speech evaluation by speech therapist (Fig. 5). The objective assessment was done to evaluate nasal emission, speech intelligibility and nasalance scores by nasometer. Report from definitive speech aid prosthesis was compared with previous reports (without prosthesis and with interim prosthesis). Marked improvement in speech quality was noticed after assessment with definitive speech aid prosthesis. The nasalance score had reduced further from $57 \%$ (with interim prosthesis) to $48 \%$ (with definitive prosthesis). Much improvement in oral speech quality was seen, from $44 \%$ (with interim prosthesis) to $38 \%$ (with definitive prosthesis) (Table 1).
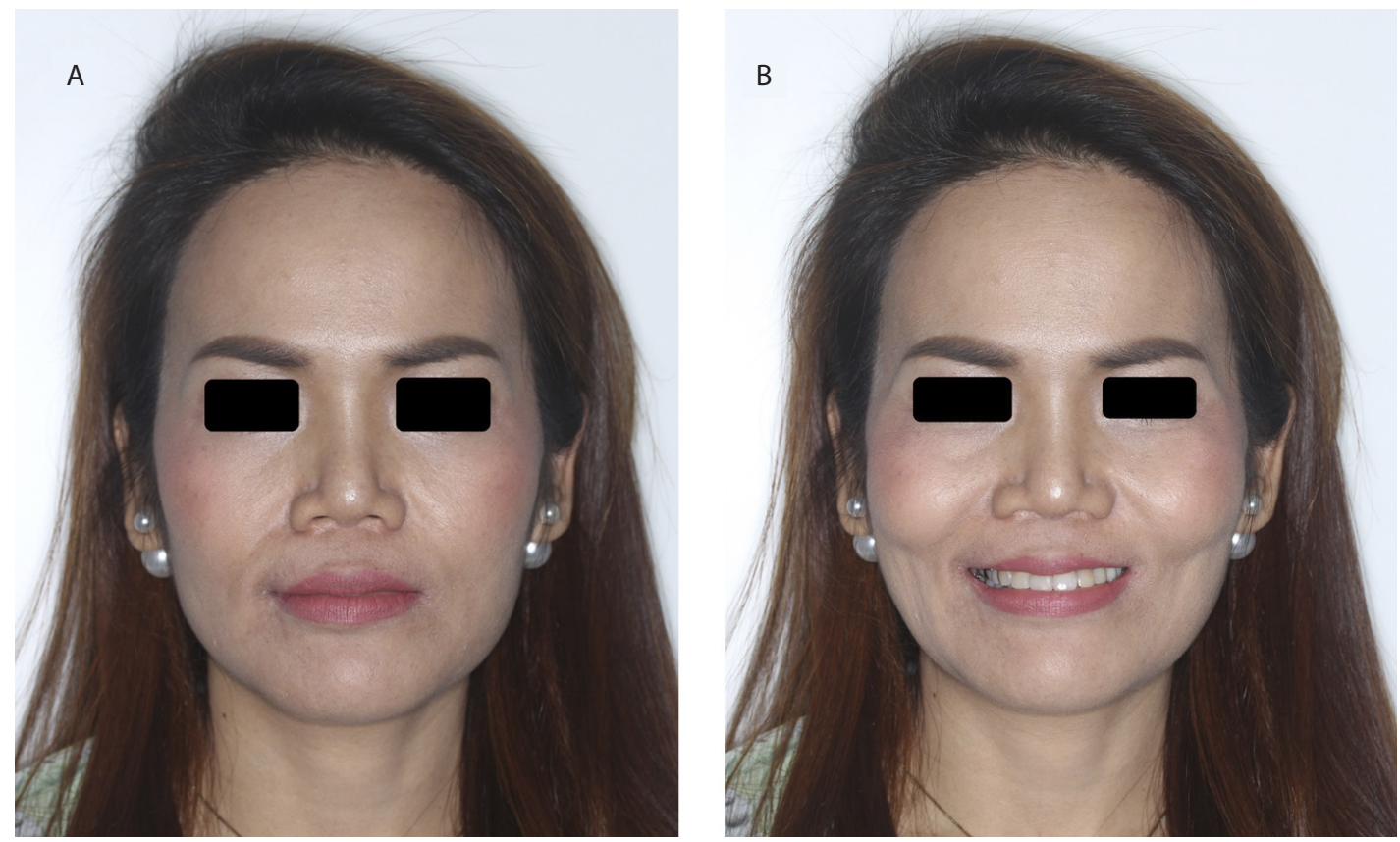

Fig. 4 Extraoral presentation with definitive prosthesis. (A) Adequate labial fullness after insertion of prosthesis. (B) Lip support and harmonious appearance during smile.
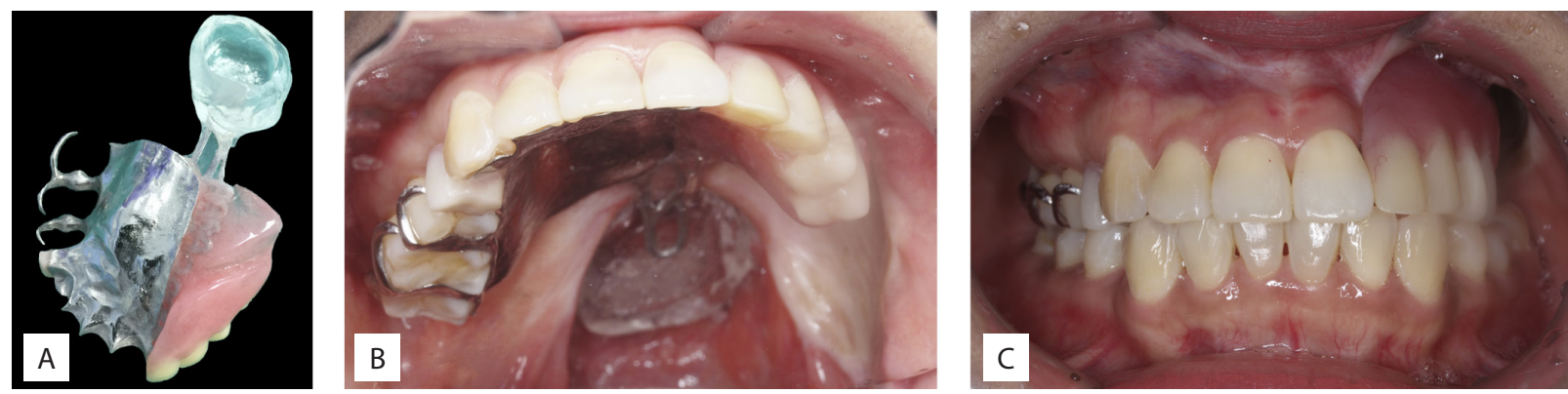

Fig. 5 Intra-oral presentation with definitive prosthesis. (A) Definitive speech aid prosthesis. (B) The inferior portion of the obturator was kept parallel to the horizontal hard palate. (C) Definitive prosthetic outcome.

Design of speech aid prosthesis fulfilling the esthetic and functional requirements. 


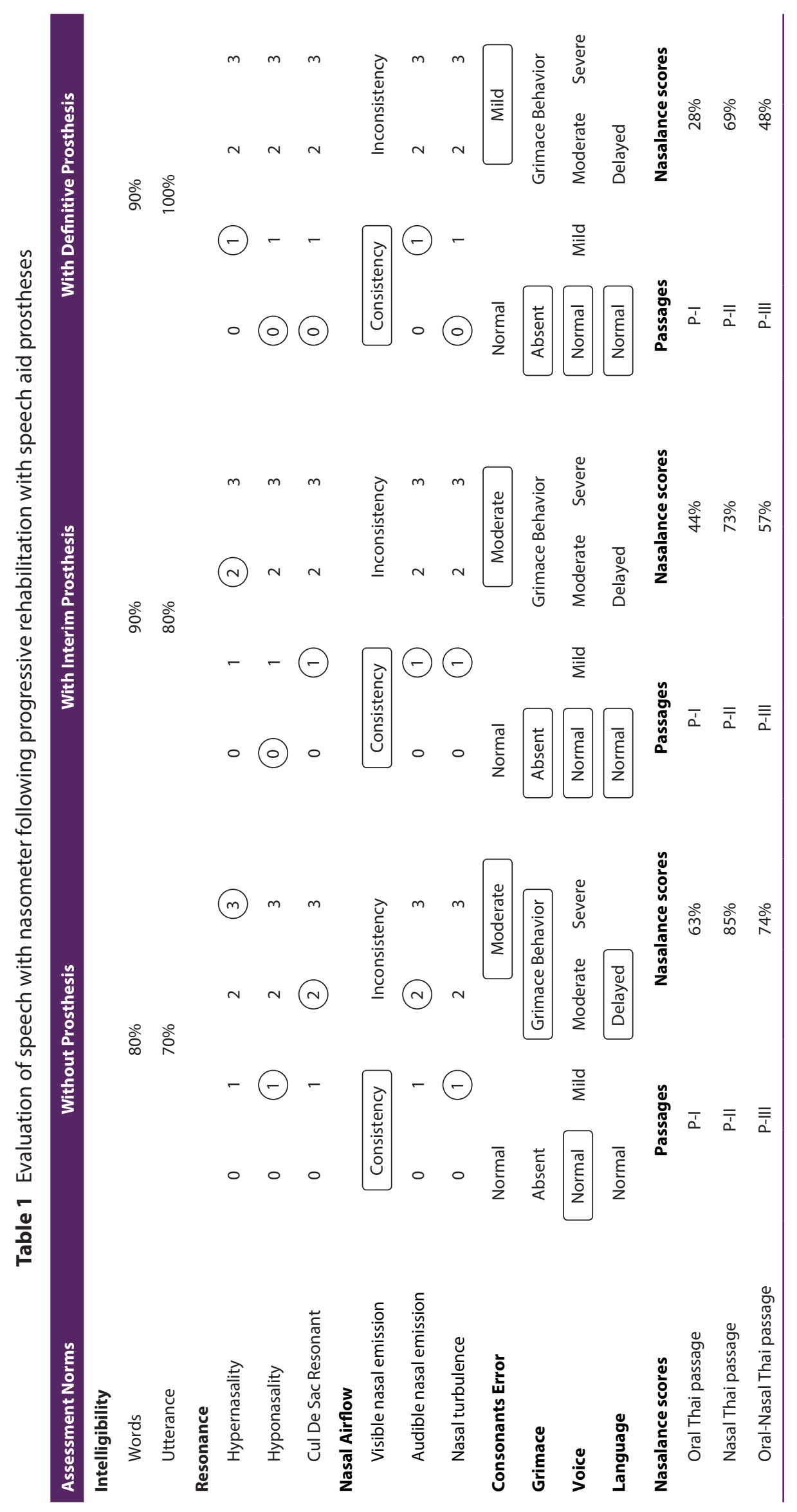




\section{Ethics Statement}

The case publication is in the scope of the permission to further use medical data signed by every patient at the hospital. The patient signed the general university consent form. This general consent was approved by the local ethics committee of the university (Ref. No: 4834412).

\section{DISCUSSION}

The impact of surgical resection of tumor varies according to the procedure, size, location and the extent of resection, and subsequently the type of reconstruction during rehabilitation procedure (Kuroda et al., 2000; Rieger et al., 2009). Velopharyngeal insufficiency defects associated with soft palate cancer resection leave the patient with psychological and functional deficiency. The soft palate deficiency leads to hypernasality and nasal emission due to leakage of air through the nose during speech (Kummer et al., 1992). This abnormal distribution of acoustic energy and air pressure within the oral cavity and nasal cavity during speech should be restored either by surgical reconstruction or by prosthetic rehabilitation procedures. The two most commonly used procedures for correction of VPI are the posterior pharyngeal flap and the sphincter pharyngoplasty. These procedures help by decreasing the size of the velopharyngeal port. Recently, few surgical techniques designed to improve velopharyngeal closure gained popularity which includes Furlow's palatoplasty and palatal re-repair (Dings et al., 2014). These two techniques work by lengthening the palate and/or otherwise tightening the levator sling, which can partially decrease the escape of air through nasal cavity during speech.

Prosthetic rehabilitation includes the use of palatal speech aid obturators. The objectives of speech bulb prosthesis are to control the escape of air through nasal cavity and nasal resonance during speech and to block the leakage of materials from the nasal passage during the process of deglutition. Hence speech aid prosthesis can satisfy the psychological, esthetic and functional needs associated with velopharyngeal insufficiency defects when surgical procedures are not deemed suitable. Thus in this case, the speech aid prosthesis improved the esthetics by restoring the missing teeth and speech function by customisation of the speech bulb portion of prostheses.

Furthermore, speech assessment after surgical or prosthetic management of VPI is important for diagnostic and therapeutic purposes, therefore speech therapists play an important role in diagnosing and helping to achieve ultimate improvement in speech. Perceptual assessment is the initial phase, which helps to diagnose the extent and impairment in speech. It is done by the examiner's assessment of speech during prescribed reading tasks. The objective assessment of speech gives a more in depth idea of speech impairment, which is done by using different devices.

The objective assessment of voice and resonance can be done by a nasometer. It provides a helpful biofeedback tool during stimulatory testing and during treatment by measuring the acoustic energy entering into the oral and nasal cavities during speech. The computerised instrument with the help of software, then calculates the proportion of oral and nasal resonance as a nasalance score. In this case, the nasometer assessment was done prior to the fabrication of speech aid prosthesis, which was compared to the nasometer reports after the fabrication of interim and definitive prosthesis in order evaluate the improvement in speech quality and resonance. The various parameters had shown improvement in speech quality, specifically the nasalance scores, which were showing a consistently decreasing trend from $74 \%$ nasalance scores without prosthesis to $48 \%$ with definitive prosthesis. Quality of oral speech had refined from $63 \%$ without prosthesis to $28 \%$ with definitive prosthesis. This improvement in speech can 
be anticipated due to appropriate speech bulb relationship to posterior pharyngeal wall and lateral wall tissues of the defect. The progressive assessment of speech not only helped to diagnose the intensity of resonance, degree of nasal emission and speech intelligibility, but further helped to improve the design of the bulb portion of speech aid prosthesis by providing the consistent feedback for nasalance scores in percentage.

\section{CONCLUSION}

Interim speech aid prosthesis helped to diagnose the speech intelligibility, resonance and consonants errors by nasometer assessment which were overcome in definitive prosthesis therefore speech aid prosthesis served the purpose of diagnostic and therapeutic aid in assessing and improving the speech intelligibility, resonance, nasal emission and nasalance scores while treating velopharyngeal insufficiency defect.

\section{ACKNOWLEDGEMENTS}

Authors wish to acknowledge the academic, clinical and laboratory support of Maxillofacial Prosthetic Service, Mahidol University Dental Hospital. No separate funding was required for this clinical case.

\section{REFERENCES}

Atik B, Bekerecioglu M, Tan O, Etlik O, Davran R, Arslan H (2008). Evaluation of dynamic magnetic resonance imaging in assessing velopharyngeal insufficiency during phonation. If Craniofac Surg, 19(3): 566-572. https://doi.org/10.1097/ SCS.0b013e31816ae746
Curtis TA, Beumer J III (1996). Restoration of acquired hard palate defects: Etiology, disability, and rehabilitation. In: Beumer J III, Curtis TA, Marunick MT (eds.), Maxillofacial Rehabilitation: Prosthodontic and Surgical Considerations. St. Louis: Ishiyaku EuroAmerica, Inc., pp. 225-284.

Dings JP, Mizbah K, Bergé SJ, Meijer GJ, Merkx MA, Borstlap WA (2014). Secondary closure of small- to medium-size palatal defects after ablative surgery: Reappraisal of reconstructive techniques. F Oral Maxillofac Surg, 72(10): 2066-2076. https://doi.org/10 $.1016 /$ j.joms.2014.04.036

Kao DS, Soltysik DA, Hyde JS, Gosain AK (2008). Magnetic resonance imaging as an aid in the dynamic assessment of the velopharyngeal mechanism in children. Plast Reconstr Surg, 122(2): 572-577. https://doi.org/10.1097/PRS $.0 \mathrm{~b} 013 \mathrm{e} 31817 \mathrm{~d} 54 \mathrm{~d} 5$

Kummer AW, Curtis C, Wiggs M, Lee L, Strife JL (1992). Comparison of velopharyngeal gap size in patients with hypernasality, hypernasality and nasal emission, or nasal turbulence (rustle) as the primary speech characteristic. Cleft Palate Craniofac $\mathcal{F}$, 29(2): 152-156. https://doi.org/10.1597/ 1545-1569_1992_029_0152_covgsi_2.3.co_2

Kuroda H, Inoue $\mathrm{K}$, Amatsu M (2000). Evaluation of speech function after mesopharyngeal reconstruction with radial forearm flap. Kobe $\mathcal{F}$ Med Sci, 46(5): 205215.

Meek MF, Coert JH, Hofer SO, GoorhuisBrouwer SM, Nicolai JP (2003). Short-term and long-term results of speech improvement after surgery for velopharyngeal insufficiency with pharyngeal flaps in patients younger and older than 6 years old: 10-year experience. Ann Plast Surg, 50(1): 13-17. https://doi .org/10.1097/00000637-200301000-00003 
Mink van der Molen AB, Janssen K, Specken TF, Stubenitsky BM (2009). The modified Honig velopharyngoplasty - A new technique to treat hypernasality by palatal lengthening. I Plast Reconstr Aesthet Surg, 62(5): 646-649. https://doi.org/10.1016/ j.bjps.2007.12.023

Pegoraro-Krook MI, Dutka-Souza Jde C, Marino VC (2008). Nasoendoscopy of velopharynx before and during diagnostic therapy. f Appl Oral Sci, 16(3): 181-188. https://doi. org/10.1590/s1678-77572008000300004

Rieger J, Bohle Iii G, Huryn J, Tang JL, Harris J, Seikaly H (2009). Surgical reconstruction versus prosthetic obturation of extensive soft palate defects: A comparison of speech outcomes. Int $\mathcal{F}$ Prosthodont, 22(6): 566572.

Seagle MB, Mazaheri MK, Dixon-Wood VL, Williams WN (2002). Evaluation and treatment of velopharyngeal insufficiency: The University of Florida experience. Ann Plast Surg, 48(5): 464-470. https://doi.org/ 10.1097/00000637-200205000-00003
Sie KC, Starr JR, Bloom DC, Cunningham M, de Serres LM, Drake AF et al. (2008). Multicenter interrater and intrarater reliability in the endoscopic evaluation of velopharyngeal insufficiency. Arch Otolaryngol Head Neck Surg, 134(7): 757-763. https://doi.org/10.1001/archotol .134 .7 .757

Sipp JA, Ashland J, Hartnick CJ (2008). Injection pharyngoplasty with calcium hydroxyapatite for treatment of velopalatal insufficiency. Arch Otolaryngol Head Neck Surg, 134(3): 268-271. https://doi.org/10 $.1001 /$ archotol.134.3.268

Tuna SH, Pekkan G, Gumus HO, Aktas A (2010). Prosthetic rehabilitation of velopharyngeal insufficiency: Pharyngeal obturator prostheses with different retention mechanisms. Eur $\mathcal{F}$ Dent, 4(1): 81-87.

Van Lierde KM, Bonte K, Baudonck N, Van Cauwenberge P, De Leenheer EM (2008). Speech outcome regarding overall intelligibility, articulation, resonance and voice in Flemish children a year after pharyngeal flap surgery. A pilot study. Folia Phoniatr Logop, 60(5): 223-232. https://doi.org/10.1159/000151242 